\title{
Hyaluronic Acid Conjugated Metformin-Phospholipid Sonocomplex: A Biphasic Complexation Approach to Correct Hypoxic Tumour Microenvironment
}

This article was published in the following Dove Press journal: International Journal of Nanomedicine

\author{
Michael M Farag' \\ Nevine S Abd El Malak ${ }^{1,2}$ \\ Soad A Yehia' \\ Mohammed A Ahmed ${ }^{1,2}$ \\ 'Department of Pharmaceutics and \\ Industrial Pharmacy, Faculty of Pharmacy, \\ Cairo University, Cairo, Egypt; ${ }^{2}$ School of \\ Pharmacy, New Giza University, Giza, \\ Egypt
}

Purpose: Development of hyaluronic acid conjugated metformin-phospholipid sonocomplexes (HA-MPS), a biphasic complexation product compiled for enhancing both the lipophilicity and targeting potential of Metformin (MET) to CD44 receptors on pancreatic cancer.

Methods: MET was chemically conjugated to hyaluronic acid (HA) via amide coupling reaction. Then, the HA conjugated MET was physically conjugated to Lipoid ${ }^{\mathrm{TM}} \mathrm{S} 100$ via ultrasound irradiation. A combined D-optimal design was implemented to statistically optimize formulation variables. The HA-MPS were characterized through solubility studies, partition coefficient, drug content uniformity, particle size and zeta potential. The optimized HA-MPS was tested via proton nuclear magnetic resonance, infrared spectroscopy to elucidate the nature of physicochemical interactions in the complex which was further scrutinized on molecular level via molecular docking and dynamic simulation.

Results: The solubility and partition studies showed a lipophilicity enhancement up to 67 folds as they adopted inverted micelles configuration based on the packing parameter hypothesis. The optimized HA-MPS showed 11.5 folds lower $\mathrm{IC}_{50}$, extra $25 \%$ reduction in oxygen consumption rate, better reduction in hypoxia-inducible factor and reactive oxygen species in MiaPaCa-2 cells.

Conclusion: These results proved better internalization of MET which was reflected by abolishing hypoxic tumour microenvironment, a mainstay toward a normoxic and less resistant pancreatic cancer.

Keywords: metformin, pancreatic ductal adenocarcinoma, hypoxia-inducible factor, MiaPaCa-2, hyaluronic acid

\section{Introduction}

Pancreatic ductal adenocarcinoma (PDAC) is one of the most aggressive and lethal malignancies facing humanity. PDAC is ranked as the fourth leading cause of cancer-related mortality and prospected to become the second by $2030 .{ }^{1}$ Although PDAC is characterized by infrequent incidences, it is the culprit behind more than 42,000 deaths in the united stated alone. PDAC has a pessimistic prognosis of 5 years survival for less than $5 \%$ of the patients which has not changed since the last 30 years. $^{2}$

Such dismal prognosis is due to the inherent resistance to chemotherapy, late diagnosis and the scarceness of efficient targeted therapy. ${ }^{3}$ Furthermore, PDAC is characterized by the presence of hypoxic microenvironment which boosts its chemoresistance leading to poor clinical outcomes. ${ }^{4-6}$ The stillness of hypoxic
Department of Pharmaceutics and Industrial Pharmacy, Faculty of Pharmacy, Cairo University, Kasr El-Aini Street,

Cairo, I 1562, Egypt

Tel +002-01228150250

Email michael.farag@pharma.cu.edu.eg
International Journal of Nanomedicine 2021:16 1005-1019

1005 
tumours render them up to 3 folds more resistant to radiotherapy and chemotherapy relative to normoxic tumours. ${ }^{7}$

Moreover, PDAC includes around 63 genetic mutations in a dozen of different signaling pathways, alongside the high desmoplastic reactions and the hypovasculature representing a challenging obstacle against chemotherapy which resulted in inadequate delivery of chemotherapeutics. ${ }^{8}$ Thus, conventional chemotherapy incapable of differentiating between normal and mutated cells will cause undesirable side effects and inefficient control on tumour progression. Therefore, there is a great need of targeted therapy to achieve better clinical outcomes. ${ }^{9}$

Metformin (MET) is one of the oldest and safest antidiabetics used in the late fifties. An expansion in the clinical indications of MET was announced to be used in polycystic ovary syndrome as well as in gestational diabetes. ${ }^{10}$ MET was spotted for cancer research back in 2010, when a report claimed that MET reduced cancer risk in diabetic patients by approximately $40 \%$ compared to other antidiabetics. ${ }^{11}$ As of April 2020, 350 clinical trials on metformin and cancer have been registered. ${ }^{12}$

The antitumorigenic properties of MET is by acting on a systemic and local levels. The systemic level is by lowering circulating glucose in case of insulin resistance thus reducing secondary hyperinsulinaemia, which will indirectly prevents the mutagenic properties of insulin. ${ }^{13}$ The local level is through mitigating the hypoxic tumour microenvironment via reducing oxygen consumption rate (OCR), inhibiting hypoxia-inducible factor (HIF-1 $\alpha$ ) and protecting DNA from damage caused by reactive oxygen species (ROS). ${ }^{14}$

The high polarity of MET - a BCS Class III drug of high solubility and low permeability - limit its cellular uptake to organic cation transporters which might reduce its use in the field of cancer therapy. ${ }^{15}$ Consequently, to reach cancerous cells in adequate concentration, MET must be administered in a hypothetically enormous doses practically unachievable in patients. ${ }^{16}$

Complexation of drugs with phospholipids - being a component of cell membrane - enhance drug permeation and cellular uptake. ${ }^{17}$ The implementation of such complexation reactions via ultrasound irradiation provides an affordable and efficacious mean for such preparations unachievable by conventional techniques. The physical concept adopted in materials synthesis is acoustic cavitation (bubbles formation, expansion and collapse) which creates peculiar conditions of temperature and pressure, tailor-made for the synthesis of nanostructured products. ${ }^{18}$
Hyaluronic acid (HA) is a glycosaminoglycan, composed of repeating disaccharide $\beta 1,3 \mathrm{~N}$-acetyl glycosaminyl- $\beta 1,4$ glucuronide. HA is a versatile compound, thanks to its many functional groups that can be easily modified and conjugated. Direct conjugation of HA offers an efficacious mean for preparing nanosystems which possess improved stability, solubility, circulation time and targeting potential since HA has a strong binding affinity to CD44 and RHAMM receptors overexpressed in many tumours including PDAC. ${ }^{19}$

The aim of this study is to prepare hyaluronic acid conjugated metformin-phospholipid sonocomplexes (HAMPS) to enhance the lipophilicity and targeting potential of MET to CD44 cell surface receptors on pancreatic cancer, in order to boost its antitumorigenic effect at physiologically achievable concentration. A D-optimal combined design was implemented to study and to optimize formulation variables. The partition coefficient and saturated solubility comparative study were performed to monitor physical changes of HA-MPS compared to MET. Molecular docking, fourier transform infrared (FTIR) spectroscopy and proton nuclear magnetic resonance ( $\left.{ }^{1} \mathrm{H}-\mathrm{NMR}\right)$ were done to scrutinize the nature of physicochemical interactions in HA-MPS. Also, molecular dynamic simulations (MDS) in water and in chloroform were done to monitor the spatial arrangement of HA-MPS when dispersed in both solvents. Finally, we compared HA-MPS and MET performance in MiaPaCa-2 cells regarding $\mathrm{IC}_{50}$, OCR, cell proliferation in presence/ absence of glucose, HIF- $1 \alpha$ and ROS to assess their ability to abolish tumour hypoxic microenvironment.

\section{Materials and Methods Materials}

Metformin hydrochloride (MET) was received as a kind gift from Marcyrl pharmaceutical industries (Cairo, Egypt). 1-(3-dimethylaminopropyl) 3-ethylcarbodiimide hydrochloride (EDC), N-hydroxysuccinimide sodium salt (NHS) were purchased from Sisco Research Laboratories Pvt. Ltd. (Mumbai, India). Sodium hyaluronate (HA) molecular weight (1500-1700 kDa) was purchased from Acros organics (Geel, Belgium). Lipoid ${ }^{\mathrm{TM}} \mathrm{S} 100$ (soybean phosphatidylcholine $\geq 94 \%$ ) was received as a kind gift from Lipoid Kosmetik AG (Steinhausen, Switzerland). Ortho-phosphoric acid (HPLC grade) was obtained from Merck (Darmstadt, Germany). N-octanol was purchased from Alpha Chemika (Maharashtra, India). Methanol, 
chloroform (HPLC grade); were purchased from SigmaAldrich Co. (St. Louis, USA). All other reagents and chemicals used were of analytical reagent grade.

\section{Preparation of HA Conjugated MET-Phospholipid Sonocomplexes (HA-MPS)}

In this work, we have adopted a D-optimal combined design generated by Design Expert ${ }^{\circledR}$ software (Version 7, Stat-Ease Inc., MN, USA) for investigating formulation variables. Three factors namely, A: MET\% (first mixture component), B: HA\% (second mixture component) and C: Phospholipid: mixture ratio were investigated as independent variables (Table 1). The polynomial equations created by the software were used for the optimization of the studied factors, in order to seek an optimized formulation based on the desirability score and the applied constraints. The partition coefficient $\left(\mathrm{Y}_{1}\right)$ was the target response (Table 1). The compositions of the prepared HA-MPS are listed in Table 2.

HA-MPS is a two-stages complex, the first stage was the conjugation of HA with MET via the formation of amide linkage between the amino group of MET and the carboxylic acid group of HA. Briefly, we added the required amount of $\mathrm{HA}$ in $20 \mathrm{~mL}$ ultrapure water in addition to equal amounts of 1-(3-dimethylaminopropyl) 3-ethylcarbodiimide hydrochloride (EDC) as an amide coupling agent and N-hydroxysuccinimide (NHS) to prevent the formation of $\mathrm{N}$-acylurea byproduct to achieve high purity product. The solution was stirred over magnetic stirrer for $1 \mathrm{~h}$ to allow activation of carboxylic acid group. After that, the required amount of MET was added and the solution was stirred overnight for the reaction to complete. $^{20}$

The next day, the second stage started by the evaporation of the solution to dryness under reduced pressure.
Then, in the same flask, we added the required amount of Lipoid in $50 \mathrm{~mL}$ of methanol and the mixture was placed in a sonicating water-bath (Ultrasonic cleaner $\mathrm{S} 30 \mathrm{H}$, Elma GmbH, Frechen, Germany) to be exposed to ultrasound waves for $1 \mathrm{hr}$ at room temperature. Consequently, methanol was evaporated under reduced pressure and the dried residues were stored in small jars inside the desiccator till nest use..$^{21,22}$

\section{Characterization of HA-MPSs} High-Performance Liquid Chromatography (HPLC) Analysis

MET was quantified adopting an isocratic reversed phase HPLC method with few modifications. ${ }^{23}$ The HPLC system includes a Shim-pack VP-ODS column 150 x $4.6 \mathrm{~mm}$, particle size: $5 \mu \mathrm{m}$ (Shimadzu, Kyoto, Japan) which was kept at $25.0 \pm 2.0^{\circ} \mathrm{C}, \mathrm{L}-7110$ pump, LaChrom D7000 integrator and UV-VIS L-7420 detector (Hitachi, Tokyo, Japan). The mobile phase is a slightly acidic binary system composed of methanol and double distilled water (10:90, $\mathrm{v} / \mathrm{v}$, respectively and $\mathrm{pH}=3$ using ortho-phosphoric acid). The analysis was performed at constant flow rate of $1.5 \mathrm{~mL} / \mathrm{min}$ and the detector $\lambda_{\max }$ was set at $233 \mathrm{~nm}$. The modified HPLC method was validated regarding its accuracy, precision, selectivity and linearity.

\section{Determination of Drug Content Uniformity in HA-MPS}

The drug content uniformity of MET in HA-MPS was determined by the aforementioned HPLC method. Briefly, the total content of MET was determined by dissolving $10 \mathrm{mg}$ of the HA-MPS in $20 \mathrm{~mL}$ (80:20 methanol: water) for $15 \mathrm{~min}$. The solution was filtered, evaporated under vacuum to dryness and reconstituted with the mobile phase. Aliquots of the reconstituted solutions were analyzed by the abovementioned HPLC method. The drug content uniformity was calculated as follows:

Table I D-Optimal Combined Design Used for the Optimization of HA-MPS

\begin{tabular}{|c|c|c|c|c|c|c|}
\hline \multirow[t]{2}{*}{ Independent Variables } & \multirow[t]{2}{*}{ Type } & \multicolumn{2}{|c|}{ Levels Investigated } & \multicolumn{3}{|c|}{ Optimized Level } \\
\hline & & Low Actual & High Actual & & & \\
\hline A: Metformin\% (MET\%) & Mixture & $10 \%$ & $90 \%$ & $10 \%$ & & \\
\hline B: Hyaluronic acid\% (HA\%) & Mixture & $10 \%$ & 4.87 & $90 \%$ & & \\
\hline C: Phospholipid: Mixture & Numerical & 1 & 5 & 4.87 & & \\
\hline Dependent Variables & \multicolumn{3}{|c|}{ Constraints } & Expected & Observed & Residual* \\
\hline$Y_{1}=$ Partition coefficient $(P)$ & \multicolumn{3}{|l|}{ Maximize } & 0.171 & 0.175 & -0.004 \\
\hline
\end{tabular}

Note: $*$ Residual $=$ expected value - observed value. 
Table 2 Composition and Characterization of the Prepared HA-MPS

\begin{tabular}{|c|c|c|c|c|c|c|c|c|c|c|}
\hline Formula & $\begin{array}{l}\text { MET } \\
\%\end{array}$ & $\begin{array}{l}\text { HA } \\
\%\end{array}$ & $\begin{array}{l}\text { PL: } \\
\text { Mix }\end{array}$ & $\begin{array}{l}\text { Content } \\
\text { Uniformity }^{a} \\
\% w / w\end{array}$ & $\begin{array}{l}\text { Partition } \\
\text { Coefficient }^{a}\end{array}$ & LEP & $\begin{array}{l}\text { Water } \\
\text { Solubility }^{a} \\
(\mathrm{mg} / \mathrm{mL})\end{array}$ & $\begin{array}{l}\text { Particle } \\
\text { Size }^{a}(\mathrm{~nm})\end{array}$ & $\begin{array}{l}\text { Zeta } \\
\text { Potential }^{\mathrm{a}} \\
(\mathrm{mV})\end{array}$ & $\mathbf{P D I}^{\mathbf{a}}$ \\
\hline RL I & 50 & 50 & 5 & $99.08 \pm 4.54$ & $0.1022 \pm 0.0087$ & 38.73 & $0.684 \pm 0.056$ & $587.25 \pm 25.10$ & $-47.75 \pm 0.49$ & $0.252 \pm 0.03$ \\
\hline RL 2 & 90 & 10 & 5 & $92.39 \pm 3.28$ & $0.0318 \pm 0.0011$ & 12.03 & $1.232 \pm 0.096$ & $426.15 \pm 11.38$ & $-37.55 \pm 1.20$ & $0.276 \pm 0.05$ \\
\hline RL 3 & 10 & 90 & I & $105.69 \pm 6.46$ & $0.0482 \pm 0.007 \mid$ & 18.26 & $0.246 \pm 0.021$ & $466.05 \pm 29.77$ & $-32.40 \pm 1.13$ & $0.288 \pm 0.03$ \\
\hline RL 4 & 30 & 70 & 4 & $98.45 \pm 6.14$ & $0.0606 \pm 0.0047$ & 22.97 & $0.466 \pm 0.019$ & $526.35 \pm 11.95$ & $-38.15 \pm 1.48$ & $0.231 \pm 0.01$ \\
\hline RL 5 & 90 & 10 & I & $92.81 \pm 4.51$ & $0.0095 \pm 0.0010$ & 3.59 & $2.961 \pm 0.134$ & $360.80 \pm 25.03$ & $-42.65 \pm 1.06$ & $0.273 \pm 0.01$ \\
\hline RL 6 & 90 & 10 & 3 & $95.98 \pm 1.30$ & $0.0166 \pm 0.0007$ & 6.27 & $1.380 \pm 0.080$ & $395.65 \pm 14.35$ & $-32.45 \pm 0.92$ & $0.227 \pm 0.01$ \\
\hline RL 7 & 30 & 70 & 2 & $101.61 \pm 8.19$ & $0.0381 \pm 0.0073$ & 14.44 & $0.608 \pm 0.022$ & $466.45 \pm 22.56$ & $-38.95 \pm 0.78$ & $0.226 \pm 0.01$ \\
\hline RL 8 & 90 & 10 & 5 & $92.14 \pm 4.33$ & $0.0267 \pm 0.0015$ & 10.11 & $0.940 \pm 0.043$ & $439.40 \pm 22.20$ & $-39.45 \pm 3.04$ & $0.260 \pm 0.01$ \\
\hline RL 9 & 90 & 10 & I & $95.76 \pm 6.32$ & $0.0097 \pm 0.0022$ & 3.67 & $3.710 \pm 0.503$ & $335.75 \pm 29.63$ & $-41.90 \pm 0.71$ & $0.287 \pm 0.02$ \\
\hline RL 10 & 50 & 50 & I & $94.21 \pm 3.97$ & $0.0067 \pm 0.0011$ & 2.53 & $1.332 \pm 0.022$ & $493.45 \pm 31.89$ & $-31.90 \pm 0.28$ & $0.260 \pm 0.03$ \\
\hline RL I I & 50 & 50 & 3 & $91.15 \pm 1.84$ & $0.0335 \pm 0.0015$ & 12.68 & $1.133 \pm 0.038$ & $533.24 \pm 28.11$ & $-37.75 \pm 0.92$ & $0.210 \pm 0.05$ \\
\hline RL 12 & 10 & 90 & 5 & $102.66 \pm 3.86$ & $0.1764 \pm 0.0125$ & 66.83 & $0.102 \pm 0.004$ & $668.65 \pm 36.70$ & $-37.75 \pm 0.78$ & $0.304 \pm 0.03$ \\
\hline RL 13 & 10 & 90 & 3 & $93.31 \pm 4.20$ & $0.1077 \pm 0.0061$ & 40.79 & $0.142 \pm 0.003$ & $586.85 \pm 23.4 I$ & $-41.15 \pm 1.06$ & $0.289 \pm 0.04$ \\
\hline
\end{tabular}

Note: ${ }^{a}$ Data represented as mean \pm standard deviation $(n=3)$.

Abbreviations: PL, phospholipid; mix, mixture.

Drug content uniformity $\%=W a / W t \times 100$

Where Wa is the actual content of MET, and Wt is the theoretical content of MET.

\section{Solubility Studies, Determination of n-Octanol/}

Water Partition Coefficient (P) and Lipophilicity Enhancement Parameter (LEP)

\section{Solubility Studies}

The saturated solubility of MET and HA-MPS were determined by adding known excess amounts of the inspected sample inside capped glass vials containing $10 \mathrm{~mL}$ of water. All vials were shaken at 100 strokes/min for 1 day at $37^{\circ} \mathrm{C}$. Consequently, the vials were centrifuged to separate the excess undissolved solids at $15,000 \mathrm{rpm}$ for 30 min. The supernatant was decanted and filtered, then an aliquot $(1 \mathrm{~mL})$ was diluted with the mobile phase to be injected in the HPLC system for quantification. ${ }^{24}$

Determination of $\mathrm{n}$-Octanol/Water Partition Coefficient (P) and Lipophilicity Enhancement Parameter (LEP)

The determination of $n$-octanol/water partition coefficient (P) and lipophilicity enhancement parameter (LEP) were done to evaluate the imparted lipophilicity on MET in HAMPS. Concisely, we added a known weight of MET or HAMPS in capped glass vials containing $10 \mathrm{~mL}$ of water and shaken for 1 day at $37^{\circ} \mathrm{C}$. Then, the solutions were centrifuged at 4,000 rpm for $15 \mathrm{~min}$ and decanted to separate the clear aqueous solution from the excess undissolved residues. Then, we added $10 \mathrm{~mL}$ of n-octanol to the clear aqueous solution to be shaken for another day at $37^{\circ} \mathrm{C}$. After that, the solutions were centrifuged at 4,000 rpm for $15 \mathrm{~min}$ in order to separate the two phases, where each phase was individually filtered through a $0.45 \mu \mathrm{m}$ membrane filter. Using the abovementioned HPLC method, the concentrations of MET in each phase to calculate the partition coefficient $(\mathrm{P})$ through following equation:

$$
\mathrm{P}=\mathrm{Co} / \mathrm{Cw}
$$

Where Co is the concentration of MET in organic phase (n-octanol), $\mathrm{Cw}$ is the concentration in aqueous phase (water). The lipophilicity enhancement parameter (LEP) was calculated through the following equation:

$$
\mathrm{LEP}=\mathrm{P} \text { of HA-MPS } / \mathrm{P} \text { of MET }
$$

\section{Measurement of Particle Size, Polydispersity Index} (PDI) and Zeta Potential

We dispersed $10 \mathrm{mg}$ of HA-MPSs per $10 \mathrm{~mL}$ of water, the solution was filtered to remove macro-aggregates and then adequately diluted (1:20) to measure the average particle diameter (z-average) and the polydispersity index (PDI) through diffraction light scattering technique at $173^{\circ}$. The zeta potential $(\zeta)$ was measured through electrophoretic analysis, using Zetasizer Nano ZS (Malvern Instrument Ltd., Worcestershire, UK).

\section{Statistical Optimization}

The optimized formula was chosen based on the desirability factor generated by Design Expert ${ }^{\circledR}$ software through 
the applied constraint on the partition coefficient as shown in Table 1. The aim was enhancing the lipophilicity of MET through sonocomplexation with phospholipid while attaching HA which is a hydrophilic targeting moiety. Then, the suggested optimized formula was prepared and evaluated to compare the actual partition coefficient with that predicted by the software.

\section{Characterization of the Optimized Formula}

\section{Proton Nuclear Magnetic Resonance ('H-NMR)}

The AVANCE III Nano Bay $400 \mathrm{MHz}$ FT-NMR spectrophotometer (Bruker, USA) was used to record the ${ }^{1} \mathrm{H}-\mathrm{NMR}$ spectra of MET, HA, Lipoid and optimized HAMPS in deuterated methanol. Chemical shift values $(\delta)$ were recorded in ppm. The purpose was to inspect the type of interactions between the HA conjugated MET and Lipoid $^{\mathrm{TM}}$ S100 besides confirming the FTIR data concerning the formation of amide linkage between MET and HA.

\section{Fourier-Transform Infrared Spectroscopy (FTIR)}

FT-IR spectra of MET, HA, HA conjugated MET, Lipoid and optimized HA-MPS were recorded by FT-IR spectrophotometer (Shimadzu, Kyoto, Japan) using potassium bromide discs. The purpose of the comparative spectrophotometric analysis was to confirm the formation of amide linkage between MET and HA as well as the sonocomplexation of HA conjugated MET with Lipoid. All samples were scanned over $4000-500 \mathrm{~cm}^{-1}$ range at room temperature. Spectral smoothing techniques and baseline correlation were implemented.

\section{Transmission Electron Microscopy (TEM)}

The morphological shape of the optimized HA-MPS after water dispersion was determined using TEM (Joel JEM 1400, Tokyo, Japan). Briefly, an aliquot of the dispersed sample was adequately diluted with water, filtered through $0.2 \mu \mathrm{m}$ syringe filter and mixed with $1 \%$ phosphotungstic acid. Consequently, a carbon-coated copper grid was impregnated with the mixture then was left at room temperature to drain off any excess and to dry to be examined using TEM. ${ }^{24}$

\section{Molecular Docking and Molecular Dynamics Simulation (MDS)}

Molecular docking was used to predict the interactions between the HA conjugated MET (HA-MET) and Lipoid. The molecular docking was divided into two steps, the first step was to draw the HA-MET using
ChemDraw $^{\circledR}$ ultra-version 19.0.0.22 (PerkinElmer informatics, inc., Waltham, MA, USA), both HA and MET structures were drawn in $2 \mathrm{D}$ form using ChemDraw ${ }^{\circledR}$ and were linked by forming amide bond between MET primary amine group and the carboxylic acid group of the D-glucuronic acid moiety of HA. Then, the 2D structure was converted to $3 \mathrm{D}$ using Chem $3 \mathrm{D}^{\circledR}$.

The second step was done using MOE version 2015.10 (Chemical Computing Group Inc., Montreal, Canada), where the HA-MET and Palmitoyl-Linoleoyl Phosphatidylcholine (PLPC the main component in Lipoid) were drawn, charges were assigned and their energy was minimized using Amber10: EHT force field to an energy gradient of $0.1 \mathrm{kcal} / \mathrm{mol}^{25} \mathrm{HA}-\mathrm{MET}$ was then docked into PLPC. The best docked module was chosen based on the binding energy scores, ${ }^{1} \mathrm{H}-\mathrm{NMR}$ and FTIR characterization results.

The molecular dynamic behaviour of one monomer of the most favorable HA-MPS obtained from the docking step was investigated in water and chloroform systems through MDS using MOE software. The simulation system was set up using Amber10: EHT force fields, we have selected a cubic box containing 2850 water or chloroform molecules, with periodic boundary conditions and dimensions of $44.7 \AA \times 44.7 \AA \times 44.7 \AA$. The non-bonded cutoff distance was set to $10 \AA$. Consequently, the energy was minimized and the system was heated by Nose-Poincare thermostat following gradual increments up to 310 K. Finally, a simulation was run for 1 nano second (ns). ${ }^{21}$

\section{MiaPaCa-2 Cell Line}

MiaPaCa-2 cell line, acquired from the American Type Culture Collection (Manassas, VA), were cultured at VACSERA (Giza, Egypt). Cells were grown in Dulbecco's modified Eagle's medium DMEM (Invitrogen/Life Technologies, CA, USA). DMEM was supplied with $10 \mu \mathrm{g} / \mathrm{mL}$ of insulin, $10 \%$ fetal bovine serum FBS (Hyclone, UT, USA) and 1\% penicillinstreptomycin. The cells were allowed to mature in an incubator at $37^{\circ} \mathrm{C}, 95 \%$ relative humidity and $5 \% \mathrm{CO}_{2}$.

Antiproliferative Assay (MTT) and Cell Proliferation in Presence/Absence of Glucose

The MTT assay is a fundamental test for the assessment of the cytotoxicity of the optimized HA-MPS. The MTT assay is based on the production of a bluish-purple formazan reduction product of 3-[4,5 dimethylthiazol-2-yl]2,5 diphenyltetrazolium bromide (MTT) by mitochondrial 
reductase enzyme in living cells. ${ }^{26}$ Stock solutions of MET (positive control) and the optimized HA-MPS in DMSO were individually diluted into serial dilutions $(0.39,1.56$, $6.25,25$ and $100 \mu \mathrm{g} / \mathrm{mL})$. A 96-well plate $\left(1.2-1.8 \times 10^{4}\right.$ cells/well) was used. Before the MTT assay, $100 \mu \mathrm{L}$ of growth medium $+100 \mu \mathrm{L}$ of the dilutions of the investigated compounds were incubated in each well for $48 \mathrm{~h}$. Then, the MTT solution was added in each well and incubated for $2 \mathrm{~h}$. the formed formazan crystals were dissolved using MTT solubilization solution (Sigma cata$\log$ no. M-8910). The cellular metabolic activity was estimated spectrophotometrically at $450 / 690 \mathrm{~nm}$ by Robonik P2000 Elisa Reader. After that, the concentration-effect relationship curves were constructed to determine the $\mathrm{IC}_{50}$ $(\mu \mathrm{g} / \mathrm{mL})$ by interpolation. The $\mathrm{IC}_{50}$ values were represented as the mean \pm standard error pooled from three experiments.

In a 96-well plate $\left(1.2-1.8 \times 10^{4}\right.$ cells/well), wells were treated individually with the $\mathrm{IC}_{50}$ concentration of HAMPS or MET and incubated for $24 \mathrm{~h}$ either in presence or absence of glucose. Subsequently, the cells were trypsinized and counted by Vi-Cell (Beckman-Coulter, Brea, CA, USA). Cell count was expressed as viability percentage employing trypan blue exclusion. ${ }^{27}$

\section{Oxygen Consumption Rate (OCR) Measurement}

The XF24 Seahorse Extracellular Flux Analyzer (Seahorse, Bioscience, North Billerica, MA, USA) was used to measure the oxygen consumption rate. In the seahorse cell plate, MiaPaCa-2 $\left(1.3-1.6 \times 10^{4}\right.$ cells $)$ were incubated overnight. After that, the MiaPaCa-2 cells were treated with the $\mathrm{IC}_{50}$ concentration of either MET or HA-MPS and incubated for $2 \mathrm{~h}$ for conditioning then the OCR was measured for $2.5 \mathrm{~h}$. The results were expressed as percentage OCR. ${ }^{27}$

\section{Hypoxia-Inducible Factor (HIF-I $\alpha$ )}

The HIF1AN ELISA Kit (MyBioSource, San Diego, USA, catalogue no. MBS9305423) was used to measure HIF-1 $\alpha$ concentration. In a 96-well plate, the plates were divided into either test, standard or control. We added $50 \mu \mathrm{L}$ of cell lysate to each well incubated with either HA-MPS, MET or sample diluent, respectively. After that, the wells were incubated for $1 \mathrm{~h}$ with $100 \mu \mathrm{L}$ of HRP-conjugate reagent at $37^{\circ} \mathrm{C}$ and wrapped with a closure membrane. Then, we washed the plates for 4 consecutive times. Subsequently, the wells were incubated with $50 \mu \mathrm{L}$ chromogen solution $\mathrm{A}+50 \mu \mathrm{L}$ chromogen solution $\mathrm{B}$ for
$15 \mathrm{~min}$ at $37^{\circ} \mathrm{C}$. Finally, $50 \mu \mathrm{L}$ stop solution was added to each well, we waited for $5 \mathrm{~min}$ to allow colour transformation from blue to yellow. Then, the optical density was measured using Robonik P2000 ELISA reader at 450 $\mathrm{nm}$. The HIF-1 $\alpha$ concentrations were obtained through converting the measured optical densities using a standard calibration curve and expressed as $\mathrm{pg} / \mathrm{mL}$. The results were expressed as mean \pm S.D. of three experiments.

\section{Reactive Oxygen Species (ROS)}

ROS detection assay Kit (Catalog no. K936-250, Biovision, California, USA) was used for the quantification of ROS. The principle of the assay depend on a fluorogenic probe (H2DCFDA), which is converted inside the cell into H2DCF (non-fluorescent) by cell esterases. Then, H2DCF is re-oxidized into a highly fluorescent product by ROS inside the cells depending on their concentration. In brief, a 96-well plate $\left(1.3-1.6 \times 10^{5}\right.$ cells/ well) was incubated with ROS inducer besides either MET or HA-MPS. The cell suspension was harvested at room temperature using centrifugation at $300 \mathrm{x} \mathrm{g}$ for $5 \mathrm{~min}$. Subsequently, we have re-suspended the cell pellets with ROS assay buffer together with ROS label and incubated for $30 \mathrm{~min}$. Then, the fluorescence was measured at 495/ $529 \mathrm{~nm}$ by Robonik P2000 ELISA reader. We have subtracted the background to determine the change in fluorescence. ${ }^{27}$

Quantification of Intracellular MET/MPS Using HPLC MiaPaCa-2 cells were incubated with the $\mathrm{IC}_{50}$ concentration of either MET or HA-MPS for $24 \mathrm{~h}$ on dishes $(10 \mathrm{~cm}$ diameter) with full growth medium. Then, the cells were harvested after washing with Dulbecco's PhosphateBuffered Saline (DPBS). Subsequently, the cells were homogenized in DPBS and extracted using methanol: methylene chloride (1:2) for 3 times. The organic layers were collected together and evaporated under vacuum. We have reconstituted the residues with the mobile phase to be quantified using the aforementioned HPLC method. ${ }^{28}$

\section{Results}

\section{Determination of Drug Content Uniformity in HA-MPS}

The range of drug content of the prepared HA-MPS was found to vary from $92.14 \% \pm 4.33 \%$ to $105.69 \% \pm 6.46 \%$, as represented in Table 2, indicating the reproducibility of the preparation technique. 


\section{Solubility Studies, Determination of} n-Octanol/Water Partition Coefficient (P) and Lipophilicity Enhancement Parameter (LEP)

The aqueous solubility of the prepared HA-MPS ranged from $0.102 \pm 0.004$ to $3.710 \pm 0.503 \mathrm{mg} / \mathrm{mL}$ as shown in Table 2. Obviously, the aqueous solubility of the prepared HA-MPS is reduced relative to pure MET (486.324 \pm $11.608 \mathrm{mg} / \mathrm{mL}$ ), this reflects the imparted lipophilicity of HA-MPS.

The results of n-octanol/water partition coefficient were in accordance with the aqueous solubility results, where it ranged from $0.0067 \pm 0.0011$ to $0.1764 \pm$ 0.0125 as shown in Table 2 relative to that of pure MET $(0.0026 \pm 8.88 \mathrm{E}-06)$, the lowest value of LEP was about 2.5 fold in formula RL 10 and the highest value was about 67 fold in formula RL 12. ANOVA results showed that the combined cubic mixture linear process model was significant and fitting for the data $\left(\mathrm{R}^{2}=0.9735\right)$.

\section{Measurement of Particle Size, Polydispersity Index (PDI) and Zeta Potential}

Particle size is a pivotal factor that affects the pharmacokinetic parameters of the formed complexes. The prepared HA-MPS formed vesicular structures in aqueous dispersion being a phospholipid-based system. The particle size of the prepared HA-MPS was ranging from $335.75 \pm 29.63$ to $668.65 \pm 36.70 \mathrm{~nm}$. Generally, tumors are distinguished by their leaky vasculature along with the presence of intercellular gaps of $100-600 \mathrm{~nm}$, thus the prepared HA-MPSs exhibited a reasonable size for cellular penetration especially with the presence of HA targeting moiety. ${ }^{29}$

The PDI values were in the range of $0.210 \pm 0.047$ to $0.304 \pm 0.027$, indicating satisfactory degree of size uniformity. The zeta potential is a crucial parameter concerning the stability of the dispersed HA-MPS. It is clear that all of the aqueous dispersions of the prepared HA-MPS exhibited a high negative value above $-30 \mathrm{mV}$, ranging from $-31.90 \pm 0.28$ to $-47.75 \pm 0.49 \mathrm{mV}$, which is related to the presence of carboxylic groups of HA imparting such anionic nature. ${ }^{30}$ This may point the reasonable stability of the dispersed HA-MPS resulting from electrostatic repulsion. ${ }^{21}$

\section{Statistical Optimization}

The Design Expert ${ }^{\circledR}$ software, based on the applied constraint on partition coefficient, suggested a formula to be prepared with a desirability $=0.967$. Then, we prepared the suggested formula to measure its partition coefficient. Consequently, we calculated the residual between the predicted and observed responses. The small residual calculated validate the optimization process. The composition of the optimized formula is shown in Table 1.

\section{Characterization of the Optimized}

\section{Formula}

Proton Nuclear Magnetic Resonance ('H-NMR)

The ${ }^{1} \mathrm{H}-\mathrm{NMR}$ spectra of MET, HA, Lipoid and the optimized HA-MPS are shown in Figure 1A-D respectively. The ${ }^{1} \mathrm{H}-\mathrm{NMR}$ spectrum of MET showed methyl protons in $\left[-\mathrm{N}\left(\mathrm{CH}_{3}\right)_{2}\right]$ represented by signals at the region 2.8-3.3 $\delta$

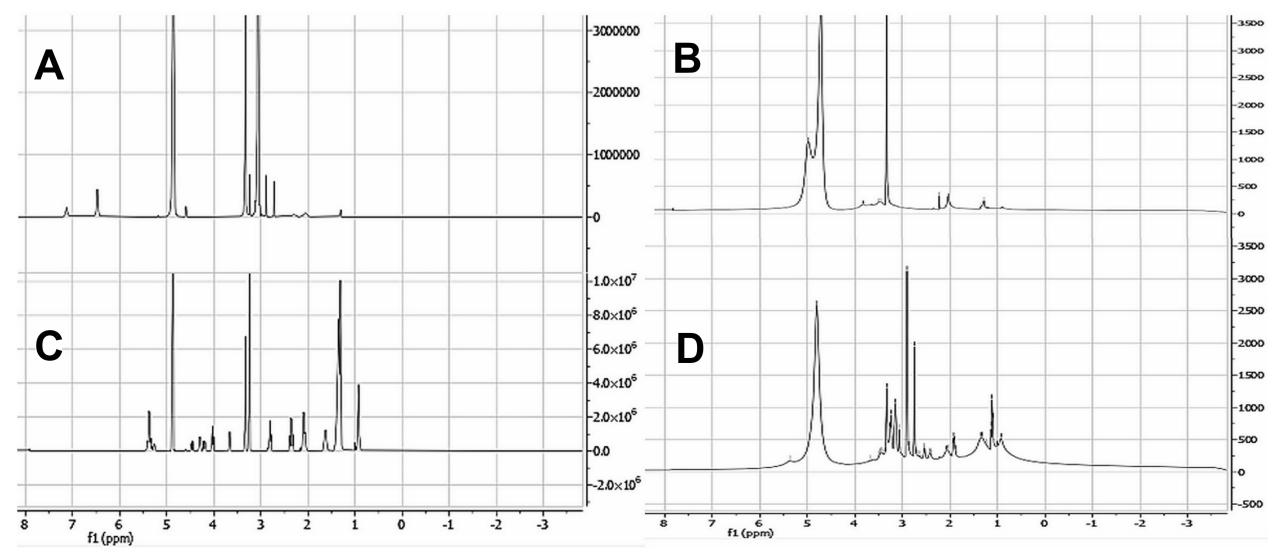

Figure I 'H-NMR spectra of (A) MET, (B) HA, (C) Lipoid and (D) optimized HA-MPS. 
(ppm), while shift values at 6.4 and $7.1 \delta$ (ppm) were attributed to amine and imines protons, respectively. ${ }^{31}$

The ${ }^{1} \mathrm{H}-\mathrm{NMR}$ spectrum of HA showed a shift value at 2-2.2 $\delta(\mathrm{ppm})$ corresponding to the acetamide moiety of the $\mathrm{N}$-acetyl D-glucosamine. The $\mathrm{CH}$ groups attached to oxygen were indicated by signals at 3.3-3.8 $\delta(\mathrm{ppm})$ and the shift value at $4.7-4.9 \delta(\mathrm{ppm})$ corresponding to protons from D-glucuronic and N-acetyl glucosamine units. ${ }^{30}$ The ${ }^{1} \mathrm{H}-\mathrm{NMR}$ spectrum of Lipoid showed the methyl protons and methylene protons of the aliphatic carbon chain represented by shift values at 0.9 and $1.3 \delta$ (ppm) respectively, protons of methyl groups in $\left[-\mathrm{N}^{+}\left(\mathrm{CH}_{3}\right)_{3}\right]$ represented by signals at $3.2-3.3 \delta(\mathrm{ppm})$, a shift value at $3.6 \delta(\mathrm{ppm})$ is attributed to the methylene protons of the choline moiety in $\left[-\mathrm{CH}_{2}-\mathrm{N}^{+}\right]$and $\mathrm{P}-\mathrm{O}$ group in $\left[-\mathrm{P}-\mathrm{O}-\mathrm{CH}_{2}-\right]$. Also, shift values at 4.1 and $5.2 \delta$ (ppm) represented methylene protons of glycerol- $\eta$ and glycerol- $\eta_{0}$ respectively. ${ }^{32}$

The ${ }^{1} \mathrm{H}-\mathrm{NMR}$ spectrum of the optimized HA-MPS showed marked changes in the appearance of shift values at $2-2.5 \delta(\mathrm{ppm})$ corresponding to the acetamide moiety of the $\mathrm{N}$-acetyl D-glucosamine, which suggest the involvement of the carbonyl group of HA in intermolecular H-bond with amino group of MET. Methylene protons of the choline moiety near $\mathrm{N}$ atoms $[-\mathrm{CH} 2-\mathrm{N}+]$ and $\mathrm{P}-$
$\mathrm{O}$ groups $[-\mathrm{P}-\mathrm{O}-\mathrm{CH} 2-]$, glycerol- $\eta$ and glycerol- $\eta_{0}$ methylene protons were shielded which suggest their involvement in H-bond with amino groups of MET, suggesting that the proximity of the HA-MET molecule caused a stronger electrical environment.

\section{Fourier-Transform Infrared Spectroscopy (FTIR)}

The FTIR spectra of MET, HA, HA conjugated MET, Lipoid and optimized HA-MPS are shown in Figure 2AE respectively. The FT-IR spectrum of MET showed the $\mathrm{N}-\mathrm{H}$ primary asymmetric and symmetric stretching vibration represented by broad absorption bands at $3371 \mathrm{~cm}^{-1}$ and $3294 \mathrm{~cm}^{-1}$ respectively, while the $\mathrm{N}-$ $\mathrm{H}$ in plane deformation appeared as a strong band at $1539 \mathrm{~cm}^{-133}$

The FT-IR spectrum of HA showed broad absorption band at $3417 \mathrm{~cm}^{-1}$ is related to the intra- and intermolecular hydrogen bond between stretching vibration of $\mathrm{OH}$ group with the stretching vibration of $\mathrm{NH}$ group. Absorption bands at $1616 \mathrm{~cm}^{-1}$ and $1411 \mathrm{~cm}^{-1}$ are belonging to symmetric and asymmetric vibration of $\mathrm{COO}^{-}$ group, respectively. Absorption band at $1045 \mathrm{~cm}^{-1}$ is corresponding to the hemiacetylic $\mathrm{C}-\mathrm{O}-\mathrm{C}$ of the saccharide unit. $^{30}$

The FT-IR spectrum of HA-MET complex showed changes in the absorption bands at $3371 \mathrm{~cm}^{-1}$ and

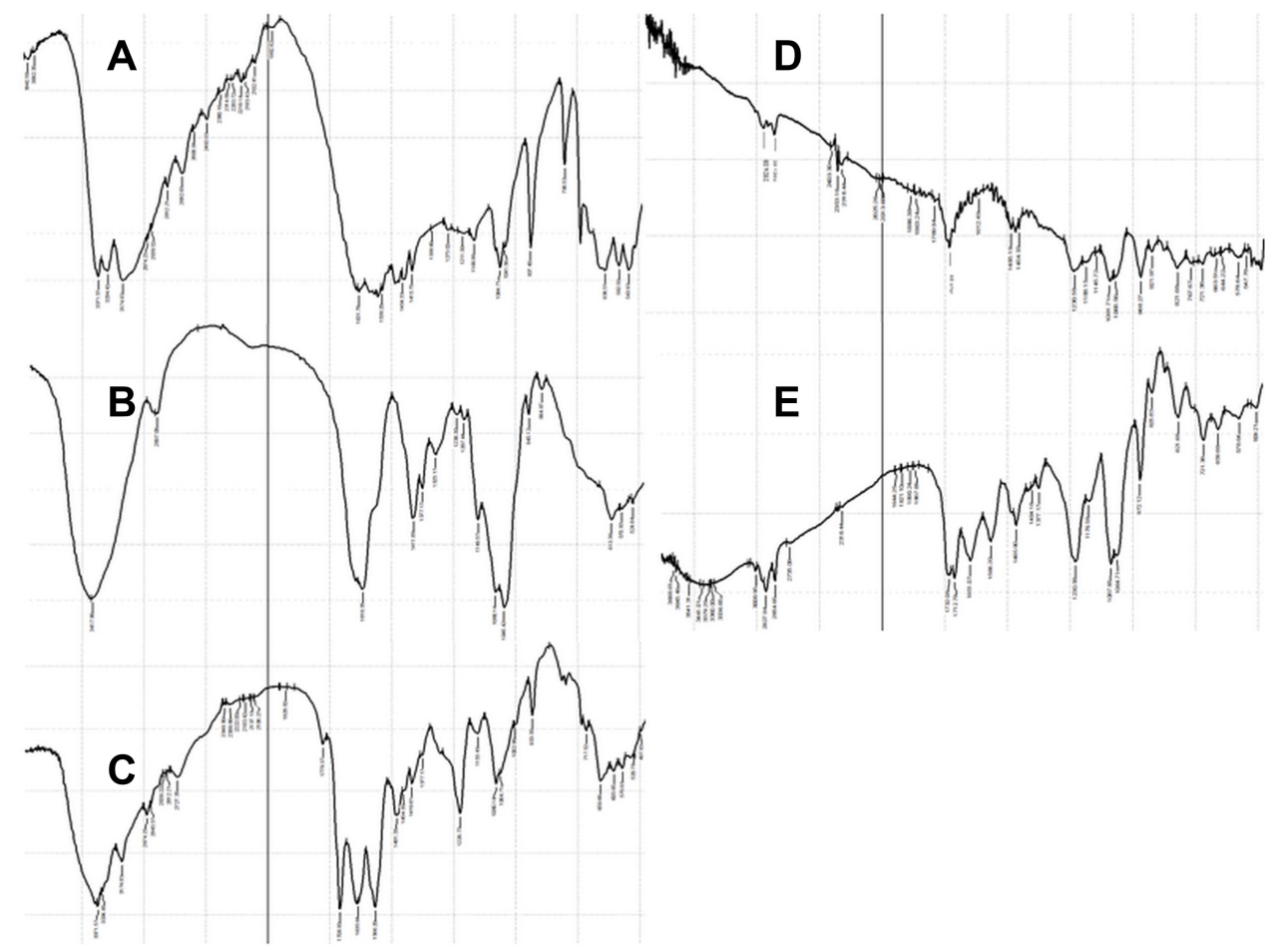

Figure 2 FTIR spectra of (A) MET, (B) HA, (C) HA conjugated MET, (D) Lipoid and (E) optimized HA-MPS. 
$3336 \mathrm{~cm}^{-1}$ belonging to the $\mathrm{N}-\mathrm{H}$ primary asymmetric and symmetric stretching vibration respectively which may infer their involvement in amide bond formation. Sharp band at $1635 \mathrm{~cm}^{-1}$ corresponding to amide bond $\mathrm{CO}-\mathrm{N}$ between the carboxylic group of $\mathrm{HA}$ and the primary amine group of MET. A strong band at $1539 \mathrm{~cm}^{-1}$ involving the $\mathrm{N}-\mathrm{H}$ in plane deformation has shifted to $1566 \mathrm{~cm}^{-1}$ this could be taken as an extra evidence for the formation of amide bond between the primary amine of MET and carboxylic acid group of HA. $^{20}$

The FTIR spectrum of Lipoid showed the C-H stretching of long fatty acid chain represented by absorption bands at $2924 \mathrm{~cm}^{-1}$ and $2854 \mathrm{~cm}^{-1}$, while a strong characteristic to the $\mathrm{C}=\mathrm{O}$ stretching band appeared at $1742 \mathrm{~cm}^{-1}$. The $\mathrm{P}=\mathrm{O}$ and $\mathrm{P}-\mathrm{O}-\mathrm{C}$ stretching bands appeared as absorption band at $1230 \mathrm{~cm}^{-1}$ and $1091 \mathrm{~cm}^{-1}$ respectively, while $\mathrm{N}^{+}\left(\mathrm{CH}_{3}\right)_{3}$ stretching band appeared at $968 \mathrm{~cm}^{-134}$

The FTIR spectrum of the optimized HA-MPS showed the masking of absorption bands at $3371 \mathrm{~cm}^{-1}$ and $3294 \mathrm{~cm}^{-1}$ belonging to the $\mathrm{N}-\mathrm{H}$ primary asymmetric and symmetric stretching vibration respectively due to their involvement in the amide bond formation with HA. The absorption band at $1742 \mathrm{~cm}^{-1}$ characteristic to the $\mathrm{C}=\mathrm{O}$ of Lipoid stretching band has shifted to $1732 \mathrm{~cm}^{-1}$ and became more prominent suggesting its involvement in the formation of H-bond with HA-MET. Sharp band at $1651 \mathrm{~cm}^{-1}$ corresponding to amide bond $\mathrm{CO}-\mathrm{N}$ between the carboxylic group of $\mathrm{HA}$ and the primary amine group of MET. Absorption band at $1230 \mathrm{~cm}^{-1}$ belonging to $\mathrm{P}=\mathrm{O}$ stretching become more sharp and prominent suggesting that it is involved in a H-bond formation. ${ }^{20}$

\section{Transmission Electron Microscopy (TEM)}

TEM micrographs of the aqueous dispersion of the optimized HA-MPS are shown in Figure 3A and B. It is clear that the optimized HA-MPS appeared in the form of vesicular structure in water with the appearance of micellar vesicles below $100 \mathrm{~nm}$.

\section{Molecular Docking and Molecular Dynamics Simulation (MDS)}

Molecular docking was implemented to reveal the nature of the interactions between HA-MET and the phospholipid. The optimized formula contained Lipoid as the

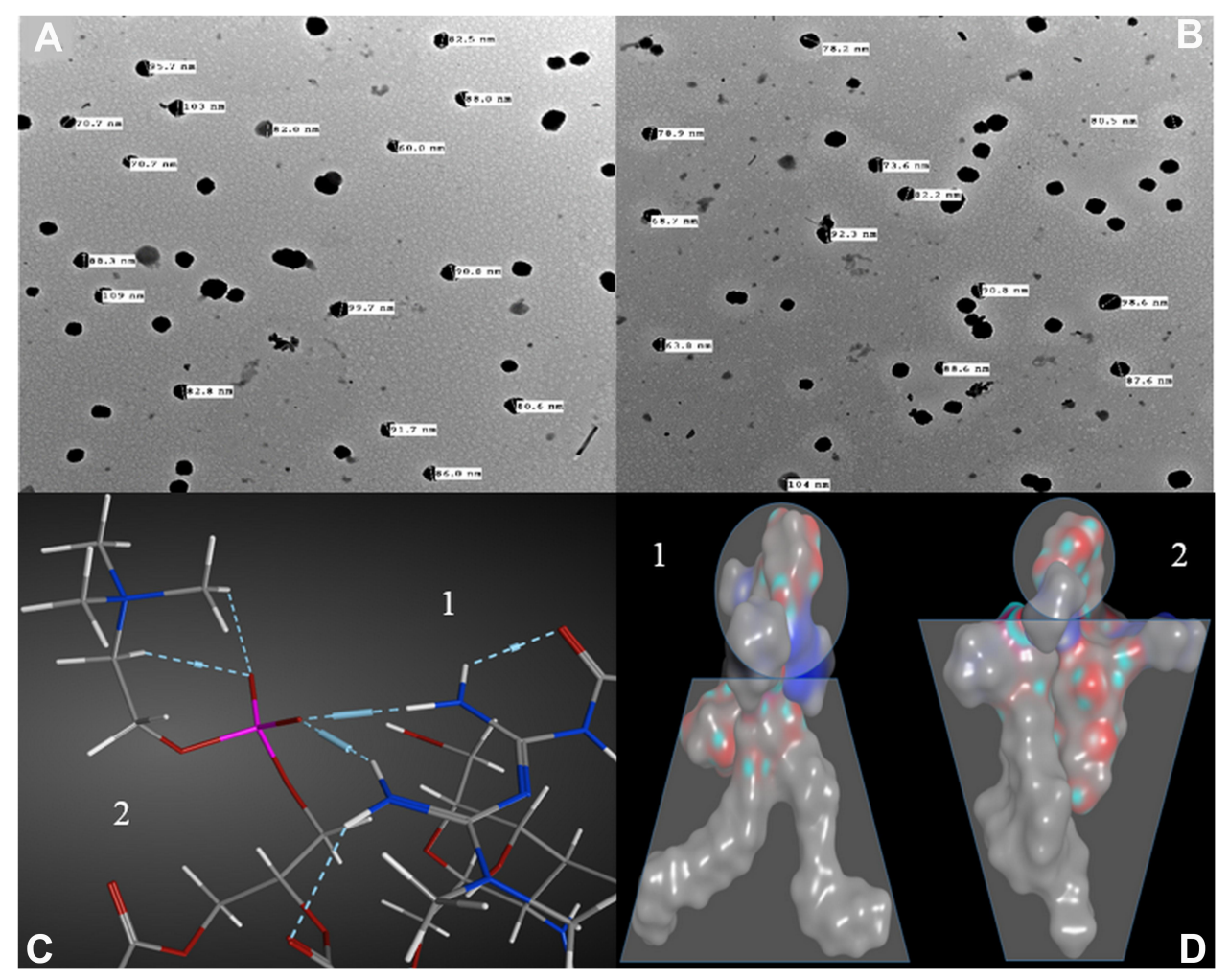

Figure 3 (A and B) Transmission electron micrographs of the optimized HA-MPS, HV= $80 \mathrm{KV}$ and direct magnification $=15000-25000 \times$ showing nanosized vesicles. (C) A snapshot of the minimum energy complex showing the interaction between (I) MET molecule and (2) PLPC polar head and non-polar tail using MOE 20I5. I0 software (D) (I) Inverted cone configuration of the optimized HA-MPS in chloroform, (2) Micellar configuration of the optimized HA-MPS in water. 
phospholipid which was represented as PalmitoylLinoleoyl Phosphatidylcholine (PLPC) the main constituent of Lipoid $^{\circledR}$ as per manufacturer data. The optimized HA-MPS was selected for the molecular docking and the following dynamics simulation studies. Figure 3C shows a snapshot of the minimum energy complex demonstrating the interaction between the HAMET molecule and PLPC. The computed energy of docking was $-7.73 \mathrm{kcal} / \mathrm{mol}$.

It can be concluded from Figure $3 \mathrm{C}$ that the amino groups of MET molecule interacted with the phosphate group of the polar head and with carbonyl group of the non-polar tail of the phospholipid molecule through strong hydrogen bond interactions, van der Waals forces and hydrophobic interactions. The results of both proton NMR and molecular docking showed a strong interaction at the polar head and non-polar tail of phospholipid.

Molecular dynamic simulations were performed to thoroughly inspect the spatial conformation adopted by the optimized HA-MPS when dispersed in water/chloroform on molecular level. Five snapshots captured at 0.2 nanoseconds interval during the simulation are shown in Figure $4 \mathrm{~A}$ and $\mathrm{B}$ respectively.
MiaPaCa-2 Cell Line

Antiproliferative Assay (MTT) and Cell Proliferation in Presence/Absence of Glucose

The MTT assay was used to determine the antiproliferative effect of the optimized HA-MPS relative to MET against MiaPaCa- 2 cells. The concentration capable of inhibiting the viability of half the cells $\left(\mathrm{IC}_{50}\right)$ was used to estimate such effect. The optimized HA-MPS $\left(\mathrm{IC}_{50}=121.48 \pm 7.39 \mu \mathrm{g} / \mathrm{mL}\right)$ had significantly $(\mathrm{P}<0.05) 11.5$ folds lower $\mathrm{IC}_{50}$ relative to MET $(\mathrm{IC} 50=1392.7 \pm 63.2 \mu \mathrm{g} / \mathrm{mL})$ as shown in Figure 5A. This was predicted based on the targeting ability of HA and the enhanced lipophilicity discussed in partition coefficient section.

Consequently, we used the $\mathrm{IC}_{50}$ concentration to evaluate the antiproliferative effect of the optimized HA-MPS and MET in presence/absence of glucose. The optimized HA-MPS showed significantly $(\mathrm{P}<0.05)$ enhanced antiproliferative effect in terms of reduced $\%$ cell viability in presence/absence of glucose $(66.39 \% \pm 2.14 \% / 57.93 \% \pm$ $2.06 \%)$ relative to MET $(95.52 \% \pm 3.84 \% / 90.02 \% \pm$ $5.17 \%$ ) as shown in Figure 5B.

\section{Oxygen Consumption Rate (OCR) Measurement}

The OCR of MiaPaCa-2 was compared after treatment with the $\mathrm{IC}_{50}$ of the optimized HA-MPS and MET. The optimized

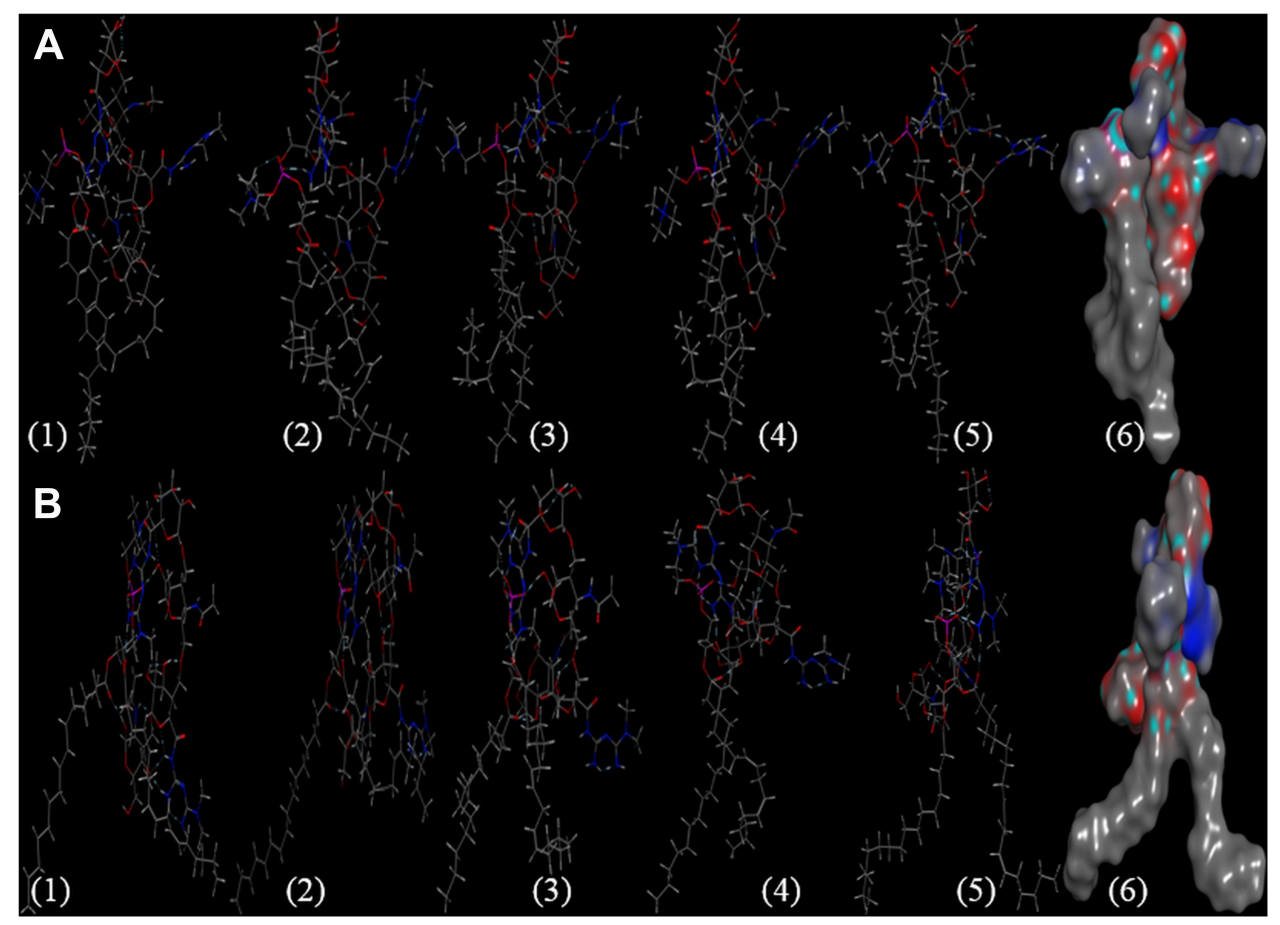

Figure 4 Snapshots from molecular dynamics simulation of the HA-MPS in water (A) and in chloroform (B), with the molecules of both solvents hidden at (I) 0.2 nanosecond, (2) 0.4 nanosecond, (3) 0.6 nanosecond, (4) 0.8 nanosecond, (5) I nanosecond and (6) 3D surface of the complex after I nanosecond using MOE 20I5.I0 software. 

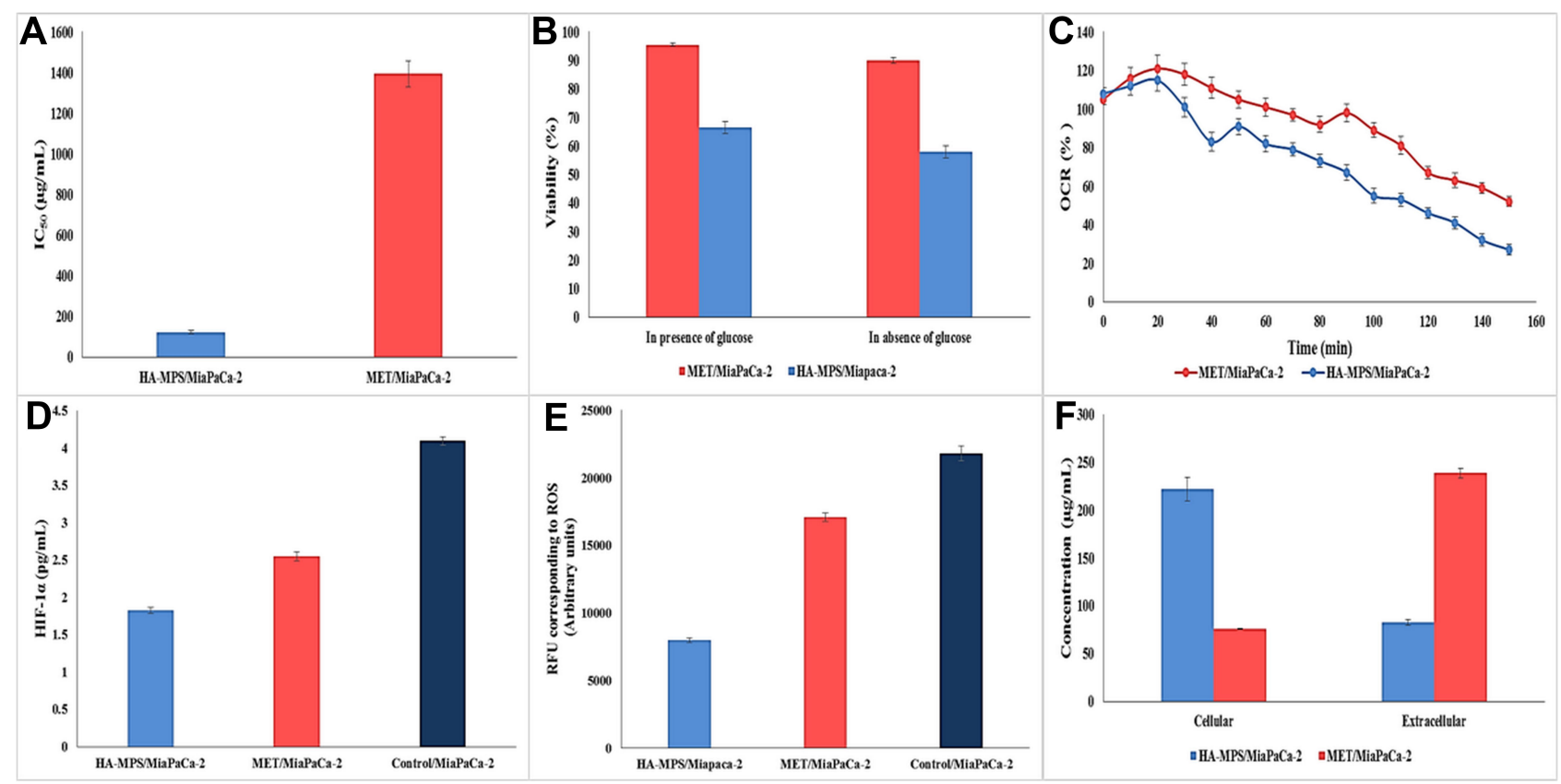

Figure 5 (A) $I_{50}$ value of the optimized HA-MPS and MET in MiaPaCa-2 cell line. (B) Percentage viability after MiaPaCa-2 treatment with either the optimized HA-MPS or MET in presence/absence of glucose. (C) Percentage OCR after MiaPaCa-2 treatment with either the optimized HA-MPS or MET. (D) HIF-I $\alpha$ level in MiaPaCa-2 cell line treated with either the optimized HA-MPS or MET. (E) Relative fluorescence units corresponding to the produced ROS after MiaPaCa-2 treatment with either the optimized HA-MPS or MET. (F) Intracellular and extracellular concentration after MiaPaCa-2 treatment with either the optimized HA-MPS or MET.

HA-MPS showed significantly $(\mathrm{P}<0.05)$ lower OCR $(73 \%)$ relative to MET (48\%) as shown in Figure 5C.

\section{Hypoxia-Inducible Factor (HIF-I $\alpha$ )}

We compared the generation of HIF-1 $\alpha$ the after treating MiaPaCa-2 with the $\mathrm{IC}_{50}$ of the optimized HA-MPS and MET as shown in Figure 5D. The optimized HA-MPS showed significantly $(\mathrm{P}<0.05)$ lower concentration of HIF-1 $\alpha(1.828 \mathrm{pg} / \mathrm{mL})$ relative to MET $(2.55 \mathrm{pg} / \mathrm{mL})$ which is compatible with OCR data. ${ }^{27}$

\section{Reactive Oxygen Species (ROS)}

As shown in Figure 5E, we compared the production of ROS after treating MiaPaCa-2 with the $\mathrm{IC}_{50}$ of the optimized HA-MPS and MET. The optimized MPS (63.43\%) showed significantly $(\mathrm{P}<0.05)$ better reduction of ROS relative to MET (21.63\%).

\section{Quantification of Intracellular MET/MPS Using HPLC} We measured the intracellular and extracellular concentration, following MiaPaCa-2 treatment with the $\mathrm{IC}_{50}$ of the optimized HA-MPS and MET, to assess the targeting potential and the imparted lipophilicity as shown in Figure 5F. The optimized HA-MPS showed significantly $(\mathrm{P}<0.05)$ higher intracellular and lower extracellular concentration $(222.46$ and $82.79 \pm 3.21 \mu \mathrm{g} / \mathrm{mL}$, respectively) compared to MET (75.53 and $238.74 \mu \mathrm{g} / \mathrm{mL}$, respectively) proving the better internalization and uptake. These findings were in accordance with the abovementioned data demonstrating the targeting potential and enhanced lipophilicity of HA-MPS relative to MET.

\section{Discussion}

MET belongs to BCS class III drugs which demonstrate high water solubility but low permeability. MET targets the mitochondria to induce cytotoxic effects in tumor cells, albeit not very effectively. Therefore, this study aimed to measure the enhancement of MET lipophilicity imparted through physical sonocomplexation with phospholipid.

The partition coefficient comparative study of the prepared HA-MPS demonstrated that HA: MET ratio showed a significant $(\mathrm{p}=0.0258)$ antagonistic interaction on n-octanol/water partition coefficient as shown in Figure 6A and B, this means that increasing HA on the behalf of MET will eventually increase partition coefficient. This could be possibly due to the formation of intramolecular hydrogen bonds between MET and HA which is expected due to the fact that HA exhibits (14 H-bond donor and $23 \mathrm{H}$-bond acceptor) while MET exhibits (3 H-bond donor and $1 \mathrm{H}$-bond acceptor). Thus, increasing HA on the behalf of MET provided the possibility to form more intramolecular H-bonds which disguised the polar groups of both compounds leading to the 

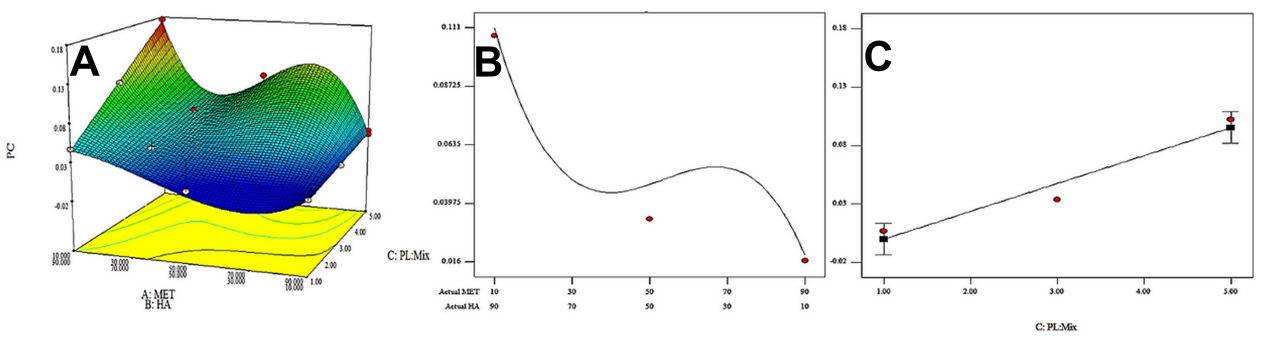

Figure 6 (A) Response surface plot of (XI: MET, X2: HA) mixture and X3: phospholipid: mixture ratio, (B) Line plot of (X1: MET, X2: HA) mixture, (C) Line plot of X3: phospholipid: mixture ratio showing their effect on the n-octanol/water partition coefficient of the prepared HA-MPS.

formation of less polar and more lipophilic conformer exhibiting higher passive lipoidal membrane permeability. ${ }^{35}$

Phospholipid: mixture ratio showed a significant $(\mathrm{p}<$ 0.0001 ) synergistic interaction with HA on n-octanol/water partition coefficient as shown in Figure $6 \mathrm{~A}$ and $\mathrm{C}$, this means that increasing phospholipid: mixture ratio from 1:1 to 5:1 while increasing the proportion of HA on the behalf of MET as abovementioned enhanced the n-octanol/water partition coefficient of MET relative to MET alone. It was previously proved that the phospholipid complexation of active ingredients enhanced their partition coefficient hence their permeability and systemic bioavailability. ${ }^{36}$ These findings are in agreement with results obtained from Rosuvastatin-phospholipid complexation where increasing phospholipid: drug ratio increased the n-octanol/water partition coefficient comparably. ${ }^{21}$

Phospholipids with high molecular weight are known for their poor aqueous solubility, so they adopt a spatial arrangement in water that shield their non-polar tails. Israelachvili et al explained the geometrical constraints that predict such assembly through calculating the packing parameter value.

The packing parameter $\left(\mathrm{v} / \mathrm{a}_{\mathrm{o}} \mathrm{l}_{\mathrm{c}}\right)$ is a fraction calculated through dividing $(v)$ the hydrocarbon chain volume by $\left(a_{o}\right)$ the surface area multiplied by $\left(l_{c}\right)$ the critical chain length. Accordingly, the shape of spherical micelles is acquired when $\mathrm{v} / \mathrm{a}_{\mathrm{o}} \mathrm{l}_{\mathrm{c}}<1 / 3$, rod-like micelles when $1 / 3<\mathrm{v} / \mathrm{a}_{\mathrm{o}} \mathrm{l}_{\mathrm{c}} \leq 1 /$ 2 or bilayered vesicles if $1 / 2<v / a_{o} l_{c} \leq 1$ and extended bilayers when $v / a_{o} l_{c}=1$. They could acquire an inverted cone configuration when $v / a_{o} l_{c}>1$, the inverted cone configuration is achieved by amphiphiles with double chains, very small head or polyunsaturated bulky chains. ${ }^{37}$

Unsaturated phospholipid like Lipoid are known to have a large hydrocarbon chain volume (v) represented by its two acyl chains. The lack of flexibility of the acyl chains is attributed to the double bonds which kept their shape rigid, atomic rotation around the double bonds is prohibited by their steric hindrance. Thus, the two acyl chains remained stretched without bending. ${ }^{38}$

In case of dynamic simulation in chloroform, as shown in Figure 3D, the carbonyl group of the acyl chains of the phospholipid formed two H-bonds, one with the amino group of MET and the other with the hydroxyl group of the N-acetyl D-glucosamine portion of hyaluronic acid. The two hydrogen bonds pulled both acyl chain apart from each other like an opened compass, thus increasing chain volume (v). At the same time, the HA-MET complex formed numerous H-bonds with polar head of phospholipid, thus keeping surface area $\left(a_{o}\right)$ small. Consequently, the optimized HA-MPS acquired an inverted cone structure based on the packing parameter theory - keeping the hydrophilic drug MET together with the hydrophilic polymer HA attached to the polar head within the hydrophilic core while the hydrophobic tail directed towards chloroform forming w/o inverted micelles. Therefore, the complexation of HAMET and unsaturated phospholipid, the packing parameter of the phospholipid alone which was normally around unity was changed into modified packing parameter value exceeding unity in chloroform. ${ }^{39}$

On the contrary, in the case of water, as shown in Figure 3D the HA-MPS acquired entirely different spatial arrangements. MET and HA being both very hydrophilic, the HA moiety stretched vertically while keeping the attached MET molecules on both sides in a horizontal manner like a cross in order to form as many H-bonds with water as possible in all directions. At the same time, the phospholipid molecule formed only $\mathrm{H}$-bonds with its polar head at the center of the cross structure and stretched vertically in a parallel manner as an extension of the HA moiety with both acyl chains close to each other as they formed only one H-bond between one carbonyl group with a hydroxyl group of the vertical HA molecule, so they were not pulled apart but they were like a closed compass to avoid contact with water molecules. 
Consequently, it acquired a micellar configuration thus keeping the hydrophilic drug MET along with the hydrophilic polymer HA towards water and very stretched horizontally while keeping the phospholipid molecule directed toward the hydrophobic core in a very tight manner to offer maximum protection of the rigid hydrophobic tail. $^{39}$

These findings are also supported by the partition coefficient and saturated solubility studies, where the partition coefficient increased about 67 fold, the saturated solubility in n-octanol increased suggesting the assembly of inverted cone structure complex into w/o inverted micelles and the saturated solubility in water decreased due to the extremely high molecular weight as well as the attachment of a lipophilic moiety leading to decreased water solubility compared to MET alone.

MET is a polar drug which is absorbed through organic cation transporters. ${ }^{40}$ The cell membrane contains phospholipid as a main component, so we wanted to test our hypothesis on the ability of sonocomplexation of MET with phospholipid along with the effect of attaching HA as a targeting moiety to target the CD44 cell surface receptors to enhance its cellular uptake and to alleviate MiaPaCa-2 cell line hypoxic microenvironment. ${ }^{19}$

Consequently, we noticed that HA-MPS required lower $\mathrm{IC}_{50}$ (11.5 folds) relative to MET which proved the enhanced cellular uptake, this was also confirmed by measuring the intracellular concentration which was tripled in case of HAMPS relative MET. Also, we found that the antiproliferative effect of HA-MPS and MET in absence of glucose is relatively better than in presence of glucose. Wheaton et al have also found similar results as they discovered that in presence of glucose MET inhibit cell proliferation while it could induce cell death when they are deprived of glucose. ${ }^{27}$

Abnormal vasculature is a common feature among solid tumours including pancreatic cancer, such abnormality caused a hypoxic microenvironment where oxygen supply is insufficient to fulfill oxygen demand. Hypoxia is the culprit behind the poor prognosis such as high recurrence chances, inefficiency of radiotherapy and metastasis. ${ }^{4}$ It is not mandatory to completely inhibit oxygen consumption to abolish tumour hypoxic microenvironment, as Grimes et al found that even $30 \%$ inhibition is capable to overcome severe hypoxia. ${ }^{41}$ Accordingly, through testing OCR we found that HA-MPS reduced OCR by $25 \%$ more than MET at even lower concentrations.

Radiotherapy acts by generation of ROS through water radiolysis, ROS generates reversible DNA radicals by attacking DNA. In presence of normoxic conditions, the DNA radicals are converted into harshly repairable stabilized DNA peroxides. Gray et al discovered the "oxygen enhancement effect" highlighting the necessity of normoxic condition in cancer radiotherapy. ${ }^{42}$

Hypoxic microenvironment induce ROS production via mitochondrial complex III which activate HIF-1 $\alpha$ to support tumorigenesis. ${ }^{43}$ Therefore, we comparatively measured the HIF-1 $\alpha$ which resulted in HIF-1 $\alpha$ concentration reduced by $28 \%$ more with HA-MPS relative to MET. OCR and HIF- $1 \alpha$ data proved the ability of HA-MPS to abolish hypoxic tumour microenvironment as a result of better cellular uptake and targeting relative to MET.

In normal cells, ROS generation cause the damage and/ or mutation of mitochondrial DNA and protein creating an endless loop that triggers cancer initiation and progression. $^{43}$ Wheaton et al revealed that MET directly inhibits complex I by acting on the flavin site in an upstream manner thus reducing ROS production, while it inhibits the electron flow from mitochondrial complex I to complex III thus indirectly reducing ROS production. ${ }^{27}$

The comparative measurement of ROS production revealed that HA-MPS reduced ROS production by 3 folds relative to MET at lower concentration. This indicates that HA-MPS might be better in preventing the initiation of pancreatic cancer as a prophylactic precaution relative to $\mathrm{MET}$.

\section{Conclusion}

In this work, HA-MPS was successfully prepared via twostage complexation technique, where we first chemically conjugated MET with HA using amide coupling reaction, then we physically complexed the HA conjugated MET with Lipoid using ultrasound irradiation followed by evaporation under vacuum. The prepared HA-MPS showed enhanced partitioning and reduced aqueous solubility relative to MET. A D-optimal combined design was implemented for the statistical optimization of formulation variables. The prepared HA-MPS showed 67 folds enhancement in the n-octanol/water partition coefficient relative to MET with reasonable particle size. MiaPaCa-2 comparative studies statistically tested by one-way ANOVA showed the significant improvement of the optimized HA-MPS relative to MET including 11.5 folds lower $\mathrm{IC}_{50}$, extra $25 \%$ reduction in OCR, 28\% extra reduction in HIF- $1 \alpha$ concentration, 3 folds lower ROS production and tripled intracellular concentration. These findings 
demonstrated the imparted lipophilicity and targeting potential of the optimized HA-MPS compared to MET.

Concisely, HA-MPS provided a versatile mean for enhancing the lipophilicity and the targeting ability of MET to abolish tumour hypoxic microenvironment effectively. An ideal chemotherapy regimen is a riddle yet to be solved, but the use of MET in such regimens might provide a closer step towards normoxic and less resistant cancers like PDAC.

\section{Disclosure}

The authors have no conflicts of interest to report in this work.

\section{References}

1. Rahib L, Smith BD, Aizenberg R, Rosenzweig AB, Fleshman JM, Matrisian LM. Projecting cancer incidence and deaths to 2030: the unexpected burden of thyroid, liver, and pancreas cancers in the United States. Cancer Res. 2014;74(11):2913-2921. doi:10.1158/ 0008-5472.CAN-14-0155

2. Siegel RL, Miller KD, Jemal A. Cancer statistics, 2016. CA: Cancer J Clin. 2016;66(1):7-30. doi:10.3322/caac.21332

3. Aslan M, Shahbazi R, Ulubayram K, Ozpolat B. Targeted therapies for pancreatic cancer and hurdles ahead. Anticancer Res. 2018;38 (12):6591-6606. doi:10.21873/anticanres. 13026

4. Dhani N, Fyles A, Hedley D, Milosevic M. The clinical significance of hypoxia in human cancers. In: Seminars in Nuclear Medicine. Elsevier; 2015:110-121.

5. Petrova V, Annicchiarico-Petruzzelli M, Melino G, Amelio I. The hypoxic tumour microenvironment. Oncogenesis. 2018;7(1):1-13. doi:10.1038/s41389-017-0011-9

6. Kumar V, Gabrilovich DI. Hypoxia-inducible factors in regulation of immune responses in tumour microenvironment. Immunology. 2014;143(4):512-519. doi:10.1111/imm. 12380

7. Higgins GS, O'Cathail SM, Muschel RJ, McKenna WG. Drug radiotherapy combinations: review of previous failures and reasons for future optimism. Cancer Treat Rev. 2015;41(2):105-113. doi:10. 1016/j.ctrv.2014.12.012

8. Jones S, Zhang X, Parsons DW, et al. Core signaling pathways in human pancreatic cancers revealed by global genomic analyses. Science. 2008;321(5897):1801-1806. doi:10.1126/science.1164368

9. Olive KP, Jacobetz MA, Davidson CJ, et al. Inhibition of Hedgehog signaling enhances delivery of chemotherapy in a mouse model of pancreatic cancer. Science. 2009;324(5933):1457-1461. doi:10.1126/ science. 1171362

10. Cicero AF, Tartagni E, Ertek S. Metformin and its clinical use: new insights for an old drug in clinical practice. Arch Med Sci: AMS. 2012;8(5):907. doi:10.5114/aoms.2012.31622

11. Giovannucci E, Harlan DM, Archer MC, et al. Diabetes and cancer: a consensus report. CA: Cancer J Clin. 2010;60(4):207-221. doi:10. 3322/caac. 20078

12. U.S. National Library of Medicine. clinicaltrials.gov. Accessed April 24, 2020.

13. Pollak MN. Investigating metformin for cancer prevention and treatment: the end of the beginning. Cancer Discov. 2012;2(9):778-790. doi:10.1158/2159-8290.CD-12-0263

14. Ashton TM, Fokas E, Kunz-Schughart LA, et al. The anti-malarial atovaquone increases radiosensitivity by alleviating tumour hypoxia. Nat Commun. 2016;7:12308. doi:10.1038/ncomms12308
15. Homšek I, Parojčić J, Dačević M, Petrović L, Jovanović D. Justification of metformin hydrochloride biowaiver criteria based on bioequivalence study. Arzneimittelforschung. 2010;60(09):553-559. doi:10.1055/s-0031-1296324

16. Pernicova I, Korbonits M. Metformin — mode of action and clinical implications for diabetes and cancer. Nat Rev Endocrinol. 2014;10 (3):143. doi:10.1038/nrendo.2013.256

17. Singh C, Bhatt TD, Gill MS, Suresh S. Novel rifampicin-phospholipid complex for tubercular therapy: synthesis, physicochemical characterization and in-vivo evaluation. Int $J$ Pharm. 2014;460 (1-2):220-227. doi:10.1016/j.ijpharm.2013.10.043

18. Pokhrel N, Vabbina PK, Pala N. Sonochemistry: science and engineering. Ultrason Sonochem. 2016;29:104-128. doi:10.1016/j. ultsonch.2015.07.023

19. Choi KY, Saravanakumar G, Park JH, Park K. Hyaluronic acid-based nanocarriers for intracellular targeting: interfacial interactions with proteins in cancer. Colloid Surface B. 2012;99:82-94. doi:10.1016/j. colsurfb.2011.10.029

20. Kumar CS, Raja M, Sundar DS, Antoniraj MG, Ruckmani K. Hyaluronic acid co-functionalized gold nanoparticle complex for the targeted delivery of metformin in the treatment of liver cancer (HepG2 cells). Carbohydr Polym. 2015;128:63-74. doi:10.1016/j. carbpol.2015.04.010

21. Abd-Elsalam WH, El-Helaly SN, Ahmed MA, Al-mahallawi AM. Preparation of novel phospholipid-based sonocomplexes for improved intestinal permeability of rosuvastatin: in vitro characterization, dynamic simulation, Caco-2 cell line permeation and in vivo assessment studies. Int J Pharm. 2018;548(1):375-384. doi:10.1016/j.jpharm.2018.07.005

22. Farag MM, Abd El Malak NS, Yehia SA, Ahmed MA. Sonocomplexation as an effective tool to enhance the antitumorigenic effect of Metformin: preparation, in vitro characterization, molecular dynamic simulation \& MiaPaCa-2 cell line hypoxia evaluation. J Drug Deliv Sci Tec. 2020;101968. doi:10.1016/j.jddst.2020.101968

23. Arayne MS, Sultana N, Zuberi MH, Siddiqui FA. Simultaneous determination of metformin, cimetidine, famotidine, and ranitidine in human serum and dosage formulations using HPLC with UV detection. J Chromatogr Sci. 2010;48(9):721-725. doi:10.1093/ chromsci/48.9.721

24. Ruan J, Liu J, Zhu D, et al. Preparation and evaluation of selfnanoemulsified drug delivery systems (SNEDDSs) of matrine based on drug-phospholipid complex technique. Int J Pharm. 2010;386 (1-2):282-290. doi:10.1016/j.ijpharm.2009.11.026

25. Case DA, Darden T, Cheatham T, et al. Amber 10. University of California; 2008.

26. Zaki NG, Mahmoud WH, El Kerdawy AM, Abdallah AM, Mohamed GG. Heteroleptic complexes of cocaine/TMEDA with some f block metals: synthesis, DFT studies, spectral, thermal, cytotoxicity and antimetastatic properties. Spectrochim Acta A. 2020;229:117938. doi:10.1016/j.saa.2019.117938

27. Wheaton WW, Weinberg SE, Hamanaka RB, et al. Metformin inhibits mitochondrial complex I of cancer cells to reduce tumorigenesis. Elife. 2014;3:e02242. doi:10.7554/eLife.02242

28. Cheng G, Zielonka J, McAllister DM, et al. Mitochondria-targeted vitamin $\mathrm{E}$ analogs inhibit breast cancer cell energy metabolism and promote cell death. BMC Cancer. 2013;13(1):285. doi:10.1186/1471-2407-13-285

29. Vachutinsky Y, Kataoka K. PEG-based polyplex design for gene and nucleotide delivery. Isr J Chem. 2010;50(2):175-184. doi:10.1002/ ijch. 201000018

30. Carneiro J, Döll-Boscardin PM, Fiorin BC, Nadal JM, Farago PV, Paula JP. Development and characterization of hyaluronic acid-lysine nanoparticles with potential as innovative dermal filling. Braz J Pharm Sci. 2016;52(4):645-651. doi:10.1590/s1984-82502016000400008

31. Shahabadi N, Heidari L. Synthesis, characterization and multi-spectroscopic DNA interaction studies of a new platinum complex containing the drug metformin. Spectrochim Acta A. 2014;12 8:377-385. doi:10.1016/j.saa.2014.02.167 
32. Ahmed MA, Al-mahallawi AM, El-Helaly SN, Abd-Elsalam WH. The effect of the saturation degree of phospholipid on the formation of a novel self-assembled nano-micellar complex carrier with enhanced intestinal permeability. Int J Pharm. 2019;569:118567. doi:10.1016/j.ijpharm.2019.118567

33. Barot BS, Parejiya PB, Patel TM, Parikh RK, Gohel MC. Development of directly compressible metformin hydrochloride by spray drying technique. Acta Pharmaceut. 2010;60(2):165-175. doi:10.2478/v10007-010-0016-9

34. Zhang K, Zhang M, Liu Z, et al. Development of quercetin-phospholipid complex to improve the bioavailability and protection effects against carbon tetrachloride-induced hepatotoxicity in SD rats. Fitoterapia. 2016;113:102-109. doi:10.1016/j.fitote.2016. 07.008

35. Shalaeva M, Caron G, Abramov YA, et al. Integrating intramolecular hydrogen bonding (IMHB) considerations in drug discovery using $\Delta \log \mathrm{P}$ as a tool. $J$ Med Chem. 2013;56(12):4870-4879. doi:10.1021/ jm301850m

36. Morsi NM, Abdelbary GA, Elshafeey AH, Ahmed MA. Engineering of a novel optimized platform for sublingual delivery with novel characterization tools: in vitro evaluation and in vivo pharmacokinetics study in human. Drug Deliv. 2017;24(1):918-931. doi:10.10 80/10717544.2017.1334719
37. Israelachvili JN, Mitchell DJ, Ninham BW. Theory of self-assembly of hydrocarbon amphiphiles into micelles and bilayers. J Chem Soc Perkin Trans II. 1976;72:1525-1568.

38. Kulig W, Pasenkiewicz-Gierula M, Róg T. Cis and trans unsaturated phosphatidylcholine bilayers: a molecular dynamics simulation study. Chem Phys Lipids. 2016;195:12-20. doi:10.1016/j.chemphyslip.20 15.07 .002

39. Israelachvili JN. Intermolecular and Surface Forces. Academic press; 2015:535-573.

40. Shitara Y, Nakamichi N, Norioka M, Shima H, Kato Y, Horie T. Role of organic cation/carnitine transporter 1 in uptake of phenformin and inhibitory effect on complex I respiration in mitochondria. Toxicol Sci. 2012;132(1):32-42. doi:10.1093/toxsci/kfs330

41. Grimes DR, Kelly C, Bloch K, Partridge M. A method for estimating the oxygen consumption rate in multicellular tumour spheroids. $J$ R Soc Interface. 2014;11(92):20131124. doi:10.1098/rsif.2013.1124

42. Gray LH, Conger AD, Ebert M, Hornsey S, Scott O. The concentration of oxygen dissolved in tissues at the time of irradiation as a factor in radiotherapy. Br J Radiol. 1953;26(312):638-648. doi:10. 1259/0007-1285-26-312-638

43. Sabharwal SS, Schumacker PT. Mitochondrial ROS in cancer: initiators, amplifiers or an Achilles' heel? Nat Rev Cancer. 2014;14 (11):709-721. doi:10.1038/nrc3803
International Journal of Nanomedicine

\section{Publish your work in this journal}

The International Journal of Nanomedicine is an international, peerreviewed journal focusing on the application of nanotechnology in diagnostics, therapeutics, and drug delivery systems throughout the biomedical field. This journal is indexed on PubMed Central, MedLine, CAS, SciSearch ${ }^{\circledR}$, Current Contents ${ }^{\circledR} /$ Clinical Medicine,

\section{Dovepress}

Journal Citation Reports/Science Edition, EMBase, Scopus and the Elsevier Bibliographic databases. The manuscript management system is completely online and includes a very quick and fair peer-review system, which is all easy to use. Visit http://www.dovepress.com/ testimonials.php to read real quotes from published authors. 